\title{
ANN-based method for olive Ripening Index automatic prediction
}

\author{
Rocco Furferi, Lapo Governi, Yary Volpe
}

\begin{abstract}
The influence of ripening degree of drupes during the harvesting period is well established in olive oil sector. A range of methods for expressing the stage of maturity of olives have been proposed in scientific literature. One of the most commonly adopted methods provides the evaluation of a Ripening Index (RI) on the basis of olive skin and pulp colour. Such an index, usually performed by a panel of experts, is indicative of the ripening degree of olive varieties and may be helpful for increasing repeatability in obtaining desired quail-quantitative characteristics of olive oil. Unfortunately, the RI evaluation technique is time-consuming, subjective (depending on expert skill) and depends on environmental conditions that may affect colour appearance of olives. This work describes a novel method for rapid, automatic and objective prediction of the Ripening Index of an olive lot. The method integrates a Machine Vision system, capable of performing a colourbased raw prediction of RI, with an Artificial Neural Network (ANN) based algorithm to refine it. Experimental results demonstrate the effectiveness of the proposed approach.
\end{abstract}

